\title{
Lexical Innovation and the Periphery of Language
}

Luca Gasparri | October 2020

Penultimate draft, final version to appear in Linguistics and Philosophy

\begin{abstract}
Lexical innovations (e.g., zero-derivations coined on the fly by a speaker) seem to bear semantic content. Yet, such expressions cannot bear semantic content as a function of the conventions of meaning in force in the language, since they are not part of its lexicon. This is in tension with the commonplace view that the semantic content of lexical expressions is constituted by linguistic conventions. The conventionalist has two immediate ways out of the tension. The first is to preserve the conventionalist assumption and deny that lexical innovations bear semantic content. The second is to dynamicize the conventionalist assumption, that is, argue that presentations of unattested expressions trigger an augmentation of the standing semantic resources of the language and instantiate content as a result of this underlying update. Building on a comparison with the production of novel onomatopoeic words, iconic pseudowords and pro-speech gestures, the paper argues that the issue is best addressed by suspending the conventionalist assumption, and describes the metasemantic implications of the claim.
\end{abstract}

\section{The Problem}

Consider the following sentence.

\section{1) [Context: the speaker is describing a basketball play]}

John was fifteen feet from the basket and tried to shaq his way to the rim.

The word "shaq" is a novel zero-derivation created by turning the proper name 'Shaq', one of the several nicknames of NBA superstar Shaquille O’Neal, into a verb. ${ }^{1}$ Even with no prior exposure to the novel verb, basketball aficionados can recover the meaning of (1) quite easily: John established a low post position and attempted to exploit his physical force to bump the defender, approach the rim, and score. Practically speaking, the interpretation of "shaq" ${ }_{(1)}$ is thus a simple task to accomplish, provided the example is presented to a rational listener with the relevant background knowledge of the game of basketball, and of how Shaquille O’Neal used to play it.

1 I am not aware of previous uses of this verbification, and apologies if anyone else should be credited for the invention. That said, I can reasonably predict that most readers will be encountering the word for the first time. Also, notational caveat: from now on, I will use expressions of form $\operatorname{word}_{\mathrm{CL}}$ to notate vocabulary items and their grammatical class, and expressions of form "word"s to notate lexical occurrences or presentations of words within sentences. Accordingly, I will use $S h a q_{\mathrm{N}}$ and $s h a q_{\mathrm{v}}$ to refer to the proper name 'Shaq' and the verb 'shaq', respectively, and "shaq" ${ }_{(1)}$ to refer to the unfamiliar presentation featuring in (1). This toy notation is not completely unambiguous, but will do the job for the purposes of this paper. 
Theoretically speaking, however, examples like (1) raise a few questions. Some such questions pertain to cognitive processing. Borrowing from the jargon of machine learning, examples like (1) call for an account of the psychological mechanisms underlying "zero-shot" lexical interpretation in humans. ${ }^{2}$ But they also raise foundational questions, as they generate a tension between two independently plausible and widely endorsed assumptions about the nature of linguistic meaning (see, e.g., the essays in Szabó (2005), Burgess and Sherman (2014), and Ball and Rabern (2018)). Call these assumptions, for brevity, Semanticity and L-Conventionalism.

Semanticity

\section{L-Conventionalism}

The semantic content instantiated by presentations of well-formed, felicitous sentences is jointly constituted by the semantic content of their word-level parts.

The semantic content of word-level sentential constituents is a function of the standing lexical conventions of the language.

Semanticity encapsulates the idea that the semantic content instantiated by presentations of well-formed, felicitous natural language sentences results from the combination of the semantic contents of their lexical constituents. By "semantic content" I mean, and will consistently mean throughout the paper, the general province of semantic facts captured by the corresponding Kaplanian (1989) notion of "content". Note that the principle refers to "presentations" of sentences. I am using this amphibious notion for two reasons. First, it does the job of signaling that the principle is making a claim about sentences-in-use rather than a claim about the standing properties of sentence and expression types. ${ }^{3}$ Second, it does so while allowing Semanticity to remain neutral on the competition between views on which the proper bearers of semantic content are token utterances, and views on which the proper bearers of content are sentence and expression types themselves qua relativized to a context of utterance: my argument should be intelligible for both positions and won't hinge on taking any particular side in this dispute. Note also the conjunction of

2 In machine learning (esp. computer vision), "zero-shot learning" refers to the processes by which a machine learns to recognize objects in an image without any labeled training data to help in the classification. Approximating a bit, "zero-shot learning" deals thus with the problem of building machines capable of emulating human-level abilities in the classification of objects belonging to a class they have never encountered before (Xian et al. 2018). Since the interpretation of "shaq" ${ }_{(1)}$ can be characterized as a "zero-shot" task in this sense, I will borrow the label as a quick reminder to the fact that in cases such as (1) listeners are faced with the task of interpreting the inaugural presentation of a word which is not part of the lexicon of the language.

3 In what follows, I will sometimes omit this qualification for concision's sake. 
"well-formed" and "felicitous" in the principle, which provisionally understands grammaticality and felicity as necessary conditions for a sentential presentation to instantiate a semantic content jointly constituted by the content of its word-level parts. This is to account for the intuition that syntactic ill-formedness (see (2)) and deficiencies in semantic consistency (e.g., the category mistake in (3)) typically prevent lexical semantic contents to coherently combine into a global sentential content.

\footnotetext{
2) * The parked car Paula.

3) \# Paula drank the car.
}

With these clarifications in sight, the business of Semanticity is straightforward enough: to assert that the semantic content instantiated by presentations of sentences complying with familiar requirements of well-formedness and felicity, is a function of the semantic contents of their lexical parts. For example, the semantic content of presentations of (4) should be a function of the contents of "Paula", (4), "parked", (4), "the" (4), and "car"

4) Paula parked the car.

L-Conventionalism, then, adds that the semantic content instantiated by presentations of lexical elements, and the way these contribute via composition to the global content of their host sentences, is determined by the character-level or "standing" lexical conventions of the language. For example, in English the lexical element $I_{\text {Pro }}$ is associated by convention to a character which ensures that non-quotational presentations of the pronoun will pick out the speaker that has produced them. Likewise, in English the word eat $t_{\mathrm{V}}$ is associated by convention to a standing semantic profile whose thematic properties prevent presentations of (5) from bearing the content that Rebecca wants to eat a pleasant location.

5) Rebecca wants to eat someplace nice.

Note that L-Conventionalism encapsulates a particularized incarnation of the overarching belief in the "conventional nature" of linguistic meaning (e.g., Lewis 1969). It concerns a specific level of linguistic meaning, i.e., semantic content, and a specific variety of conventions, i.e., the specialized conventions of semantic character we would expect to be part of the lexical component of a linguistic grammar. The claim is that the semantic content instantiated by presentations of word- 
level expressions rests on these specialized linguistic conventions, and that presentations of lexical units instantiate semantic content as a function of these character-level conventions. To avoid confusion, note also that L-Conventionalism is not trying to claim that conventions of standing word meaning are the only thing lexical semantic content is a function of: it merely asserts that conventions of standing word meaning are going to feature among the resources that, possibly together with other factors (e.g., general worldly facts and features of the non-linguistic context), endow presentations of lexical expressions with semantic content. ${ }^{4}$ Furthermore, in committing to the dependence of semantic content on specialized conventions of linguistic character, LConventionalism remains agnostic on the role of non-linguistic conventions in the constitution of non-semantic meaning, such as the role of social conventions in the recovery of speaker-meaning or pragmatically enriched utterance meaning. L-Conventionalism is not the claim that conventions of linguistic character suffice to account for each and every aspect of communication, nor is it the claim that understanding the contextual meaning of the utterances we hear doesn't typically involve reasoning and inference-making taking place outside the grammar. ${ }^{5}$ It is, rather, the more specific claim that the semantic content and the compositional features instantiated by presentations of word-level expressions is mediated by the specialized body of linguistic conventions fixed in the lexical system of the language.

Now, the conjunction of Semanticity and L-Conventionalism is unproblematic if measured against presentations of sentences containing familiar words. Take (6).

6) Many students eat apples.

Semanticity argues that the semantic content of (6) should be constituted by the semantic contents of "many"((6), "students"(6), "eat"((6), and "apples"((6). L-Conventionalism adds that the semantic content of "many"(6), "students", $(6)$, "eat"( ${ }_{(6)}$, and “apples" ${ }_{(6)}$ should be a function of the standing

4 The exact scope of the set of supplementary factors that intervene in the determination of semantic content will, of course, vary depending on the theory of meaning one is assuming. A minimalist à la Borg (2004), a contextualist à la Recanati (2010), and a relativist à la MacFarlane (2014), are likely to endorse substantially different accounts of what formal processes and what resources, besides the conventions of the language, endow sentences and expressions with content. However, the matter is orthogonal to the specific claims made by L-Conventionalism and Semanticity, versions of which I take to be widely shared among parties to the debate on the nature of meaning. I'm making this explicit to stress that the two principles, and the tension between them we will consider in a moment, are not the outcome of partisan metasemantic premises, and should make sense across the spectrum of theories of meaning available on the philosophical market.

5 See, e.g., Gasparri and Murez (2019). Back to this in due course. 
semantic features associated by convention to many $y_{\mathrm{D}}$, student $t_{\mathrm{N}}, e a t_{\mathrm{V}}$, apple $_{\mathrm{N}}$, and the plural suffix $-s$ in the lexicon of English. So far, so good. However, recall (1) and John's attempt to "shaq" his way to the basket. On Semanticity, (1) should instantiate a semantic content resulting from the combination of the content of its lexical parts. L-Conventionalism adds that the lexical parts of (1) should instantiate semantic content relative to the lexical conventions of English. The problem is: $s h a q_{\mathrm{V}}$ is not in the lexicon of English. As a consequence, Semanticity and L-Conventionalism cannot be embraced simultaneously in the analysis of (1). Either the semantic content borne by (1) is constituted by the semantic content of "shaq" ${ }_{(1)}$, as per Semanticity, and the case speaks against LConventionalism. Or, because $s h a q_{\mathrm{V}}$ is not part of English, and assuming L-Conventionalism, "shaq" (1) does not bear semantic content, and (1) is a counterexample to Semanticity.

We can provide a generalized formulation of the problem. Assume, with Semanticity, that wellformed, felicitous sentences instantiate a semantic content jointly constituted by the semantic contents of their lexical parts. Assume also, with L-Conventionalism, that the instantiation of semantic content by word-level expressions is a function of the standing semantic features associated by convention to those expressions in the lexicon of the language. The combination of these two assumptions is typically straightforward, but runs into troubles as soon as it is projected onto presentations of well-formed, felicitous sentences featuring lexical innovations. ${ }^{6}$ Either lexical innovations bear semantic content and, by falling outside the conventional resources of the language, provide counterevidence to the conditions for the instantiation of semantic content set by L-Conventionalism. Or L-Conventionalism sets a genuine requirement for the instantiation of semantic content, and we should reconsider the claim that presentations of unattested words may bear full-blooded semantic content.

This paper has two goals: a programmatic goal and a substantive one. The programmatic goal is to interface the philosophical discussion on meaning and conventions with recent advances in linguistic theory on the semantics of "peripheral" devices of communication like iconic words, iconic pseudowords and gestures (e.g., Kendon 2004; Lascarides and Stone 2009; Ebert and Ebert 2014; Schlenker 2018; 2019), and suggest that a combined investigation of these apparently distant domains can lead to fruitful insights into the foundations of meaning and semantic properties. The substantive goal is to use the parallel to argue for a non-conventionalist diagnosis of the tension between Semanticity and L-Conventionalism.

6 Throughout the paper, I will use labels such as "lexical innovations", "novel expressions", and "unattested words" almost interchangeably, but always to designate presentations of expressions which are not part of the lexicon of the language. Labels like "lexical innovation" are ambiguous between (at least) two readings: one referring to the use of brand new expressions (like "shaq", $(1)$ ), and one referring to semantically unfamiliar or deviant uses of attested expressions. These are distinct objects of analysis, and this paper is about the former. 
The plan is as follows. Section 2 describes the view that the presentations of lexical innovations like "shaq" (1) trigger an implicit update of the lexical conventions of the language, which in turn allows the unfamiliar word to instantiate semantic content. Section 3 draws a parallel between (1) and sentences featuring presentations of novel onomatopoeic words, iconic pseudowords, and prospeech gestures, and uses the comparison to argue that the dynamic-conventionalist view suffers from difficulties. Section 4 presents an alternative: the view that lexical innovations motivate a metasemantics licensing the instantiation of semantic content without the mediation of the standing semantic resources of the language. Section 5 recapitulates and makes some concluding remarks.

\section{Dynamic Conventions}

Armstrong (2016) provides a nice formulation of the challenges that conventionalist approaches to meaning encounter faced with examples featuring, like (1), presentations of unattested expressions. ${ }^{7}$ Borrowing from his argument, the conventionalist has two immediate ways out of the tension between Semanticity and L-Conventionalism.

The first way out is to keep the current formulation of L-Conventionalism and give up Semanticity. Since L-Conventionalism dictates that lexical semantic content is a function of the standing lexical resources of the language, and since no $s h a q_{\mathrm{V}}$ is found in the lexicon of English, we should withdraw from the premise that "shaq" (1) may bear full-blooded semantic content. Pace Semanticity, which would predict that the semantic content of (1) should be participated in by the semantic content of "shaq" ${ }_{(1)}$, the presentation is semantically gappy at the position occupied by the zero-derivation, and "shaq" ${ }_{(1)}$ makes no compositional contribution to the content of its host sentence. If anything, it can only contribute to the communicated or "inferred" meaning of (1) thanks to general pragmatic and conversational principles.

As Armstrong (2016: 95-96) himself observes, this option does not seem particularly attractive. To start, the sentence dos not trigger the judgments of ungrammaticality or infelicity we would expect if the presentation suffered from a genuine semantic deficiency. Another issue is that in the absence of semantic content, it is unclear on the basis of what kind of pragmatic or conversational principles "shaq" ${ }_{(1)}$ might yield communicated meaning, since the usual story is that pragmatically determined word meanings require the prior availability of a semantic input to inferential comprehension. Third, and perhaps more importantly, even on first exposure the novel zero-

7 Among the examples he considers: "Bea managed to houdini her way out of her cell"; "A local resident expressed concern that incoming developers were going to east village her neighborhood in Brooklyn"; "The delivery boy managed to porch the newspaper at every house on the block"; "Pat made sure to whisky the punch before the teachers arrived". In what follows, I will keep relying on (1) as my central case study, but the rest of the argument would apply equally well to any of these other examples. 
derivation word appears to be structurally integrated to the rest of the sentence, which would be hard to explain without the instantiation of compositionally efficacious content. The point can be appreciated by considering variants of (1) where the zero-derivation successfully occurs under negation or under a modal verb. See (7) and (8).

7) John did not shaq his way to the rim.

8) You couldn't shaq to the hoop if you weren't a big man.

Likewise, borrowing from the classic tests for telicity (Dowty 1979), the novel zero-derivation seems to immediately rule out the combination of perfective form and interval adverbials. Compare (9) to (10).

9) John shaqqed to the hoop for an hour.

10) John shaqqed to the hoop in an hour.

Taken together, these observations make a plausible case that we are best advised looking for a solution that maintains the premise that "shaq" ${ }_{(1)}$ makes a genuine semantic contribution to (1).

The second way out is to keep Semanticity and revise the formulation of L-Conventionalism in such a way as to make it neutralize the tension. ${ }^{8}$ On this view, "shaq" (1) does bear semantic content, and the correct diagnosis of the tension is that we should render L-Conventionalism more flexible. The reasoning is the following. Cases like (1) challenge the doctrine of L-Conventionalism only if the specialized body of conventions of character governing the instantiation of semantic content is understood as a static inventory. But that seems an unlikely assumption. The arsenal of semantic conventions in force in a language is adaptive, ever changing; when the right conditions are in place, it can evolve abruptly, if not instantaneously (think of baptisms). The observation suggests a surprisingly simple solution to the tension generated by (1): drop the assumption that the semantic content of a lexical innovation like "shaq" ${ }_{(1)}$ can be constituted only by the conventions of semantic

8 A reviewer points out that another solution would be to assume that "shaq" (1) is a code for a definite description built with attested lexical material, something like "John tried to do the kind of thing Shaq usually does", and that "shaq" (1) inherits its content from definite description it replaces. This is certainly a possibility, but the move raises some questions. What non-ad hoc motivations do we have to believe that "shaq" ${ }_{(1)}$ is a proxy for a definite description? What would be the exact format of the description involved, and how would it fit into the original structure of (1) ("John was fifteen feet from the basket and tried to do the kind of thing Shaq usually does [his way] [?? to the rim]")? Furthermore, while the move is available for (1), it does not appear to be an open option for some of the instances of lexical innovation we will consider below. I therefore will not consider this possibility further. 
meaning holding in the language prior to the presentation of (1). In other words, the lesson we should learn from the tension is not that we should suspend Semanticity, but rather that examples like (1) motivate a dynamic reinterpretation of L-Conventionalism. Call it, for brevity, Dynamic LConventionalism.

Dynamic L-Conventionalism The semantic content of word-level sentential constituents is a function of the standing lexical conventions holding or established on the fly in the language.

Dynamic L-Conventionalism appears broadly plausible. There certainly are attractive features to the idea that following even a single instance of successful coordination on the meaning of an unattested word, the participants to a conversation can establish a semantic convention for the novel term and, e.g., expect that the conversational participants will assign subsequent presentations of the expression content consistent with this background convention. In general, this revised formulation promises to improve dramatically on its static parent when it comes to giving a promising convention-based explanation of the dynamics of general language change (Bybee 2015), lexical change (e.g., Ludlow 2014), or metalinguistic negotiation (Plunkett and Sundell 2013). The thesis also reconciles, at least in intent, familiar Davidsonian objections to conventionalist theories of meaning (see Davidson 1984; 1986; 1991) with the received picture that communication is essentially a matter of shared linguistic conventions. ${ }^{9}$ If viable, Dynamic L-Conventionalism would give us an ideal result: it would allow us to preserve Semanticity, maintain a role for specialized linguistic conventions in accounting for the semantic properties of lexical innovations, and restore the lost harmony between our assumptions at the cost of a minimal revision to the original formulation of L-Conventionalism.

However, how successful really is Dynamic L-Conventionalism? First of all, we need to be clear on an issue of scope: whether or not the principle should be expected to capture, besides the dynamics occurring after the zero-shot episode of interpretive coordination on an unattested term, also the dynamics underlying the emergence of semantic content at the inaugural presentation of a

9 It might be worth recalling that for Davidson malapropisms and cases of accidental mispronunciations were as challenging for a conventionalist theory of meaning as cases like (1). Yet, the generalization is debatable. For example, Predelli (2010) and Lepore and Stone (2017) argue that malapropisms (e.g., "The police apprehended two auspicious individuals") can be reconciled with assumptions in the same ballpark as L-Conventionalism by hypothesizing that the sentence actually presented in such cases is the one featuring the word the speaker attempted to articulate (i.e., "The police apprehended two suspicious individuals"), and that the utterance inherits the conventional semantic features of the sentence it was intended to externalize. Cf. Armstrong (2016: fn. 17). 
sentence like (1). We can, accordingly, distinguish two readings of the principle: a "weak" reading and a "literal" reading. The weak option is to evaluate Dynamic L-Conventionalism under a restriction excluding the inaugural instantiation of semantic content from the explanatory scope of the principle. In this case, Dynamic L-Conventionalism would be a claim about presentations of unfamiliar terms that have at least one salient lexical precedent. The literal option is to take the formulation of Dynamic L-Conventionalism at face value: since the principle is trying to tell us something about the "semantic content of word-level sentential constituents", and "shaq" (1) is a word-level sentential constituent, the principle should provide explanatory purchase on the inaugural presentation of (1). On this reading, the success of Dynamic L-Conventionalism hinges on its ability to capture the emergence of lexical semantic content both after and at the event of the inaugural presentation of a lexical innovation like "shaq" ${ }_{(1)}$. Importantly, only the literal reading commits to the notion that Dynamic L-Conventionalism should be retracted if the instantiation of semantic content by the inaugural presentation of "shaq" ${ }_{(1)}$ cannot be explained in conventionalist terms. On the weak reading, by contrast, no such implication is drawn: proof that the instantiation of semantic content by the inaugural presentation of "shaq" (1) is not amenable to the conventionalist account would pose no harm to Dynamic L-Conventionalism.

In what follows, I will focus on the literal reading of Dynamic L-Conventionalism. The choice has a simple reason: only the literal construal of Dynamic L-Conventionalism stands a chance of truly neutralizing the tension with Semanticity. If the premise that the inaugural presentation of "shaq" ${ }_{(1)}$ bears semantic content is correct, the tension with L-Conventionalism cannot be resolved merely by insisting that linguistic conventions can be established sometimes very quickly after an initial episode of coordination on the meaning of an unattested term. Plausible as this might be, even if the zero-shot coordination on the meaning of "shaq" ${ }_{(1)}$ did contribute to the rapid emergence of a convention generating semantic content for non-inaugural presentations of the unfamiliar term, the semantic properties instantiated by that inaugural presentation would still be a negative instance to the principle, and recreate the original tension. ${ }^{10}$ In other words, we cannot consider Dynamic LConventionalism a formally adequate answer to our problem unless we are serious about the idea that the principle manages to carve out a space for specialized linguistic conventions in the factors that determine semantic content for the first presentation of a lexical innovation.

10 For example, Armstrong's (2016) argument that a dynamic approach can preserve a role for linguistic conventions in accounting for the semantic properties of lexical innovations, seems to be an argument in favor of what I have called "weak Dynamic L-Conventionalism". Proof that weak Dynamic L-Conventionalism is viable would be of consequence, and Armstrong does a remarkable job of showing the interest and the explanatory virtues of the view. However, if I am correct, the less ambitious reading of Dynamic L-Conventionalism would not settle the specific tension between Semanticity and L-Conventionalism we are considering. 
So let us focus on literal Dynamic L-Conventionalism. Once Semanticity and literal Dynamic L-Conventionalism are combined, the zero-shot presentation of (1) entails what follows.

\section{Lexical Adaptation}

i. The speaker produces (1) and "shaq" ${ }_{(1)}$ is inaugurally presented.

ii. The presentation of "shaq" ${ }_{(1)}$ feeds the language with a novel proto-convention fixing the standing meaning of $s h a q_{\mathrm{v}}$.

iii. "shaq" (1) bears semantic content as a function of the standing features of $s h a q_{\mathrm{v}}$.

Before we dig into the prospects of Lexical Adaptation, three clarifications. The first clarification is that, as it stands, Lexical Adaptation is conditional on an orthodox metasemantics on which sentence and expression types bear speaker-insensitive standing properties, rather than a framework on which the actual subject matter of semantic theorizing is the idiolect of individual speakers. Inquiry into whether the zero-shot presentation of "shaq" (1) suffices by itself, as the combination of Semanticity and literal Dynamic L-Conventionalism suggests, to determine facts about the conventional standing features of the lexical item shaq (facts that, of course, may be altered due to subsequent negotiation by the linguistic community) presupposes that expression types are (or can be) associated to standing semantic properties which hold, and bear normative force, irrespective of their appreciation by the individual speakers. I will grant this assumption. Though I expect a suitably modified variant of my argument to hold even under the premise of an idiolect-centered metasemantics, ${ }^{11}$ keep in mind that much of what I am going to be saying makes immediate sense only through the lenses of the opposite hypothesis. ${ }^{12}$

The second clarification is that although I have appealed and will appeal, for convenience, to the notion that the linguistic conventions fixing the standing properties of lexical items are part of the "lexicon", Lexical Adaptation is not necessarily committed to a formal grammar featuring an autonomous lexical component. The claim is simply that zero-shot "shaq" (1) bears semantic content because its presentation amounts to the proposition of an "augmented" variant of English assigning $s h a q_{\mathrm{v}}$ conventional semantic features. The assessment of this claim should be meaningful and coherent both for theories on which lexical conventions are the centerpiece of an autonomous lexicon, and for frameworks on which these resources are spread across other components of the

11 Which many fear is inevitably going to suffer from major drawbacks: among others, it will flirt with a controversial form of Humpty Dumptyism, and will have a hard time making sense of how speakers may be ignorant or confused about the semantic properties of the expressions of their language (e.g., Barber 2001).

12 Thanks to an anonymous reviewer for pressing me on this. 
grammar, such as the syntax-all-the-way-down approach of Distributed Morphology (Halle and Marantz 1993; 1994) or psychological models à la Elman (2009).

The third clarification is about a point which is implicit to the setup of the argument, but nonetheless worth insisting upon. It concerns the explanatory niche of our discussion and its encapsulation from neighboring debates in philosophy of language and linguistics. The question we are after has essentially to do with the mechanisms allowing lexical innovations to instantiate compositionally efficacious content irrespective of the absence of relevant conventions of meaning. The subject has multiple connections with topics like the nature of linguistic understanding and the dynamics of communicative success and coordination (both in general and in cases featuring lexical innovations). Yet, it remains fundamentally distinct from them. Our focus is not on the nature of the interpretive mechanisms triggered in interpreters upon exposure to a sentence like (1); nor is it a clarification of the conditions that have to obtain to justifiably claim that the listener "has understood (1)", or that speaker and listener have achieved "communicative coordination" on the meaning of (1) (all questions contingent on the settling of antecedent controversies about the nature of "understanding" and "communicative coordination"). Our focus is on the factors in virtue of which inaugural presentations of unattested words such as the novel zero-derivation of (1) may instantiate semantic content, and on whether these factors, as posited by Lexical Adaptation, have to contemplate an underlying change in state of the lexical resources of the language. To put it a bit crudely, this is "metaphysics of meaning", not an armchair reconstruction of the functional architecture of language processing, nor an inquiry into the epistemology of communication. It does not deal with how speakers do or should come to an appreciation of semantic content faced with the task of interpreting (1), but with the grounds in virtue of which presentations of (1) bear the semantic properties they bear in the first place. ${ }^{13}$

Back to Lexical Adaptation. If you believe that a foundational theory of semantic content should ultimately be cashed out in conventionalist terms, Lexical Adaptation appears a very good way of meeting the challenge presented by (1). Treating presentations of lexical innovations as updates to the standing resources of the language seems to give us a framework where the evidence in favor of Semanticity and the metasemantic program of L-Conventionalism can join forces without any either-or lurking in the background. Notwithstanding this attractive feature, I believe there are reasons to cast doubt on the idea that Lexical Adaptation is the best way of approaching the odd predicament cases like (1) put us in. Dynamic L-Conventionalism may well be correct on the overarching point that linguistic conventions are flexible bodies, and that the lexical-semantic apparatus of a language can be updated sometimes very rapidly by a community of speakers. Even

13 Thanks to two anonymous reviewers for inviting me to clarify this. 
so, Lexical Adaptation is probably not the best story we can tell about how the inaugural presentation of "shaq", ${ }_{(1)}$, despite the absence of $s h a q_{\mathrm{V}}$ in the lexicon, might comply with Semanticity. I will make my case for a different strategy. On the resulting proposal, the lesson we should draw from (1) is not that we should buy into the marriage of Semanticity and Dynamic LConventionalism giving rise to Lexical Adaptation, but that we should loosen the stricture on the admissible grounds of semantic content set by L-Conventionalism, and drastically relax our metasemantics in anti-conventionalist direction.

\section{Widening the Landscape}

Let us start with two observations. The first observation, actually a programmatic reminder, is that a meaningful analysis of the tension surfacing from (1) should not lose sight of the richness and the productivity of the lexicon. It is widely accepted that lexical entries are informationally complex entities which impose various requirements on the structures in which they find themselves. Think of argument structure and of the distributional phenomena grouped under the notions of S-selection and C-selection in textbook linguistic theory (e.g., Fromkin 2000). But most importantly, the lexicon is a generative engine (a.o., Bauer 2001; Jackendoff 2002), and conventions of word meaning can be generated compositionally (Higginbotham 1986; Larson and Segal 1995). Suppose English* is an impoverished duplicate of English whose lexicon contains an entry for the prefix out-, an entry for the verb populate, and the inflectional suffix $-s$, but does not feature an entry for outpopulate $_{\mathrm{v}}$. Suppose also a speaker of English* encounters for the first time the sentence "India outpopulates Japan". Even in the absence of an entry specifying the conventional properties of outpopulate $_{\mathrm{V}}$ in English*, the lexical resources available to the speaker of English* will be perfectly sufficient to determine that the sentence features a complex inflected verb made of three familiar constituents (the prefix out-, a root borrowed from populate, the inflectional suffix $-s$ ), combine the semantic features of these constituents, and generate semantic content for the unfamiliar word. To be sure, these generative mechanisms do not help account for cases of word coinage involving semantically "primitive" lexical innovations which, like "shaq" ${ }_{(1)}$, cannot be analyzed as the child of conventionalized lexical parents, and are not generated via mechanisms that ensure semantic productivity. That said, keep in mind that the lexicon is considerably more productive than a mere list of conventional form-to-denotation pairs.

The second, more at-issue observation can be initially stated as follows. Assume Semanticity: “shaq" (1) instantiates semantic content and (1) is not semantically gappy at the position occupied by the novel zero-derivation. Now, the idea that the presentation of (1) entails an underlying augmentation of the lexical resources of English does offer a formally consistent picture of the 
grounds of (1)'s content, and does reconcile Semanticity with L-Conventionalism. But it does so at the cost of a rather cumbersome explanatory epicycle. To understand the point, consider what a listener might plausibly be required to do to interpret (1) on first exposure. Once the grammatical class and the inflectional characteristics of "shaq" ${ }_{(1)}$ have been identified, and once the interpretive system has isolated the bits of information that are relevant to produce a hypothesis about the action designated by "shaq" (1), why would a listener need to update their lexicon with an entry for shaqv to fill the semantic content of the sentence, instead of using this "grab-bag" (Rayo 2013) of information directly to formulate a hypothesis about content? While it is hard to think of the zeroshot interpretation of (1) without contemplating, at some stage, the inspection of the body of factual knowledge possessed by the listener about the style of play of Shaquille O'Neal, ${ }^{14}$ the mediation of a proto-convention of meaning for $s h a q_{\mathrm{V}}$ seems inessential to an explanation of how a listener might have epistemic access to "shaq" (1)'s content. Psychologistic concerns aside, Lexical Adaptation does offer a handy way of generalizing the baseline story set by L-Conventionalism to the realm of sentences featuring novel words. But at this point we cannot take it as antecedently evident that our way out of the tension should be a chapter of L-Conventionalism, especially if, to remain within the standard doctrine, we have to buy into the somewhat mysterious narrative that the inaugural utterance of "shaq" (1) magically enriches the language with the semantic resources required to make its content a function from a Kaplan-style character. Parsimony would probably suggest a different diagnosis. The question, thus, is whether there are independent reasons to believe that the content instantiated by the zero-shot presentation of "shaq" ${ }_{(1)}$ should be mediated by the augmentation in lexical resources posited by Lexical Adaptation.

Suppose one insists that these independent reasons have to do with the productive role that the morphology plays in the generation of the novel zero-derivation. After all, "shaq" (1) is a presentation of the verbal counterpart of a known vocabulary item $\left(S h a q_{\mathrm{N}}\right)$, and switching grammatical class while keeping word form constant is a function which takes in a lexical entry and outputs another lexical entry. Once, the rejoinder might continue, we accept the seemingly uncontroversial premise that "shaq" (1) is the occurrence of a verb derived from a preexisting name though a process of change in grammatical class, it stands to reason to think that the zero-shot presentation of (1) requires the prior enrichment of the lexicon with an entry for $s h a q_{\mathrm{v}}$ via morphological conversion,

14 As a matter of fact, cooperative lexical innovation tends to rely on an implicit theory of the informational resources required to interpret the unattested word, and on an assumption that such resources are available to the listener. For example, the production of (1) is likely to occur modulo a tacit belief that the addressee is familiar with Shaquille O'Neal and associates him to the properties that are relevant to the interpretation of "shaq" ${ }_{(1)}$ (being physically imposing and bumping defenders under the rim) in an almost stereotypical fashion. 
and that the standing semantic features associated on the fly to this lexical entry fix in turn the semantic content of the unfamiliar occurrence.

My reply comes in two steps. The first step is to note that even if we granted that the morphological rejoinder is effective for (1), it faces an issue of generality. Lexical Adaptation is supposed to give us a general model of how unfamiliar words might be able to bear semantic content despite the absence of lexical resources specifying their conventional interpretation in the language. However, words coined on the basis of morphological conversion are just one instance of lexical innovation, and the rejoinder does not extend to cases where the novel word is not generated based on familiar lexical material. Lexical innovations that have no vocabulary-level parent and are introduced by inference on properties in the domain of sensory cognition, such as novel onomatopoeic verbs, offer an especially instructive comparison. Consider (11).

11) Mary saw the girls jump on their motorbikes and schwoom through the alley.

Listeners exposed for the first time to (11) appear able to identify a strong candidate meaning for the novel verb simply by analyzing its observable form and the sentential environment where it occurs. Suppose we grant Semanticity: the sentence is felicitous and well-formed, and "schwoom" ${ }_{(11)}$ bears semantic content. What grounds the instantiation of semantic content in this case? Lexical Adaptation would answer that the ground is given by the underlying augmentation in lexical resources of the language entailed by the very act of presenting (11). Again, this story seems unnecessarily complex: it is unclear why one should ground the instantiation of semantic content on an underlying change in state of the standing resources of the language when facts about the linguistic environment of the word, its morphosyntactic features, and its observable form combined are perfectly adequate to do the job. But unlike (1), the introduction of the new verb of (11) does not rely on the conversion of any preexisting lexical material, so the augmentation in lexical resources posited by Lexical Adaptation cannot, in this case, be justified on the basis of morphological considerations of the kind we have contemplated for (1).

The second step of the reply is to note that morphosyntactic considerations are unlikely to lend support to Lexical Adaptation irrespective of the specifics of cases like (1) and (11). Suppose we grant that since the presentation of "shaq" ${ }_{(1)}$ involves morphological conversion, the production of (1) requires the prior enrichment of the lexicon with an entry for $s h a q_{\mathrm{v}}$. Suppose also we grant that while this reasoning is unavailable in the case of (11), the observation that in the presentation of (11) "schwoom" ${ }_{(11)}$ bears the syntactic features of an infinitive is everything we need to justify the prior enrichment of the grammar with $s c h$ ooom $_{\mathrm{V}}$, since an infinitive form has to be generated from 
some root lexical entry. However, there is a difference between claiming that the morphosyntactic content instantiated by "shaq" ${ }_{(1)}$ and "schwoom" ${ }_{(11)}$ requires the presence in the lexicon of the root entries $s h a q_{\mathrm{V}}$ and $s c h$ woom, and the claim that the semantic content of "shaq" (1) and "schwoom" is a function of the standing semantic features associated to those root entries. The fact that the production of "shaq" ${ }_{(1)}$ involves morphological conversion and that "schwoom" ${ }_{(11)}$ bears morphosyntactic content, might as well require the prior transition to a grammar featuring the entries $s c h$ woom $_{\mathrm{V}}$ and $s h a q_{\mathrm{V}}$. But this is no guarantee that in the process these entries will also be associated with standing semantic features yielding content for "shaq" ${ }_{(1)}$ and "schwoom" ${ }_{(11)}$. In other words, even if we grant that the morphosyntactic content of "shaq" ${ }_{(1)}$ and "schwoom" function of the standing morphosyntactic features of $s h a q_{\mathrm{V}}$ and $s c h w o o m_{\mathrm{V}}$, this is no evidence that the semantic content of the presentations is a function of the character-level semantic features of those two entries. A more parsimonious diagnosis would take "shaq" ${ }_{(1)}$ and "schwoom" ${ }_{(11)}$ to be generated relative to some proto-enrichment of the lexical system with two entries, $s h a q_{\mathrm{v}}$ and $s c h w o o m_{\mathrm{V}}$, which bear the standing features requires to fix the morphosyntactic content of the two presentations, but are devoid of any character-level meaning, and therefore unfit to play any part in the determination of their content. On this line of thinking, it would then be natural to contemplate the hypothesis that the semantic content of "shaq" ${ }_{(1)}$ and "schwoom" ${ }_{(11)}$ is generated as a function of factors sitting entirely outside the standing semantic resources of the language (e.g., in the case of (11), facts about observable morphosyntactic features of the novel presentation, facts about its sentential environment, and facts about the observable form of the occurrence). Bottom line: it is still unclear why the content instantiated by sentences like (11) and (1) should rest on the underlying emergence of a convention of standing meaning for the respective lexical innovations.

Let us pause for a moment and reconsider the situation. At the beginning of our discussion, we identified two immediate ways the conventionalist could iron out the tension between Semanticity and L-Conventionalism: strip "shaq" ${ }_{(1)}$ of semantic content and retract Semanticity, or allow "shaq" ${ }_{(1)}$ to get content relative to a tacit update in the body of conventions of standing meaning holding in the language, as per literal Dynamic L-Conventionalism cum Lexical Adaptation. We have seen that the simple tests in (7)-(10) give credence to the hypothesis that "shaq" ${ }_{(1)}$ does bear semantic content, thus giving motivations to keep Semanticity in the picture. Now, the instantiation of semantic content on behalf of a word-level part of a sentence normally requires the availability of a convention of character in the language. Since full-blooded, compositionally efficacious semantic content can only be determined relative to a convention of character, we have grounds to generalize L-Conventionalism to lexical innovations and resolve the tension through the assumption of a rapidfire change in the arsenal of conventions of the language. This seems to be the critical juncture: if 
we bought into the notion that facts about semantic content and the compositional role of the lexical parts of a sentence can be entirely determined by facts outside the semantic component of the grammar, we would fall, so to speak, into metasemantic anarchy. We would except to the orderly principle that presentations of an expression can bear full-blooded semantic content, and participate in sentential composition, only if that expression is associated to standing semantic-compositional features in the lexicon of the language (otherwise, it can serve at the best the purpose of conveying communicated content). Making this exception is a costly move; that is why we should be happy to revise L-Conventionalism and add the epicycle leading to Lexical Adaptation, despite the perplexities and the overall shadow of convolution the move seems to cast.

With all this in mind, consider (12) and (13).

12) Great. Now Mark's mood will [iconic pseudoword: [u]-SOUND DESCENDING IN PITCH]..

13) Great. Now Mark's mood will [pro-speech gesture: HAND WITH PALM UP + ARM DESCENDING DIAGONALLY]. ${ }^{15}$

We mentioned that (11) cast doubt on Lexical Adaptation because it presented a case where, due to the iconic properties of "schwoom" ${ }_{(11)}$, the instantiation of semantic content was likely to occur without the mediation of the standing semantic resources of the language. This lent circumstantial support to the hypothesis that (1)'s compliance with Semanticity could be captured through similar means. (12) and (13) present a strengthened variant of the same theoretical predicament. Once again, the meaning of the pro-speech gesture and of the pseudoword are plausibly grounded on facts outside the purview of the semantic conventions of English, due to their iconic nature. It seems fairly natural to assume that the content they contribute to the sentences they are embedded in, is generated directly by the combination of sentential context with the physical makeup of the sign, rather than by some intermediate augmentation of the conventional resources of the language. ${ }^{16}$ The

15 Note on the terminology: pro-speech gestures are gestures that replace entire words. They are distinguished from co-speech gestures, which are produced simultaneously with the spoken words they modify, and from post-speech gestures, which follow the expressions they modify.

16 Since I am putting weight on the parallels between lexical innovations and novel iconic presentations, note that I am not assuming, or trying to convince the reader, that the interpretation of iconic presentations has no place for conventions. First of all, some iconic presentations are completely conventionalized and require the knowledge of an explicit signaling convention to be successfully interpreted (another basketball analogy: think of the hand gesture used by NBA officials to signal a travel violation). Other iconic presentations rely on implicit or natural representational conventions which subjects can identify and parse even with no explicit learning, but remain "conventions" in the full sense of the term: think of Greenberg (2013) on the conventions of linear projection in pictures, and Cumming, Greenberg and Kelly (2017) on the conventions of viewpoint coherence in film. However, 
additional peculiarity of these examples is the non-linguistic nature of [u]-SOUND DESCENDING IN PITCH $_{(12)}$ and HAND WITH PALM UP + ARM DESCENDING DIAGONALLY(13). While the instantiation of semantic content by the innovation of (11) could, in principle, be made sense of through the idea of an underlying change in state of the lexical resources of the language (the issue was that the move seemed stipulative and could be ockhamized), Lexical Adaptation looks like a nonstarter when it comes to capturing (12) and (13), since the pseudoword and the gesture and are not the kind of signs that can be registered as lexical types and be associated to standing meaning by way of linguistic convention. Yet, both the pseudoword and the gesture fit the label "word-level sentential constituents" featuring in Dynamic L-Conventionalism; we would find them as terminal lexical nodes in a representation of the syntactic structure of the respective sentences. If so, we obtain a direct argument to deliver a negative verdict on Dynamic L-Conventionalism, and in turn conclude that dynamic reformulation of L-Conventionalism does not help rescue the conventionalist principle from the problem of lexical innovation.

I see three possible reactions to the pressure from (12) and (13). The first reaction would be to neutralize the pressure by claiming that $[\mathrm{u}]$-SOUND DESCENDING IN $\operatorname{PITCH}_{(12)}$ and HAND WITH PALM $\mathrm{UP}+\mathrm{ARM} \mathrm{DESCENDING} \mathrm{DIAGONALLY}_{(13)}$ are just codes for familiar words. On this view, [u]-SOUND DESCENDING IN PITCH(12) and HAND WITH PALM UP + ARM DESCENDING DIAGONALLY(13) are just presentations of the phrases "descend" or "drop down" under an atypical guise, and their contribution to the meaning of the respective sentences is determined by the conventional resources of the grammar, consistent with the original formulation of L-Conventionalism.

This line of reasoning would effectively neutralize the pressure, but conflicts with evidence that pro-speech gestures and iconic pseudowords convey gradient iconic information that cannot be emulated with standard lexical items (Schlenker 2019). For example, the modulation of the angle and the amplitude of the movement of the arm in (13) can be used to trigger fine-grained inferences about the speed and the magnitude of the change in Mark's mood ("Mark will slowly go from neutral to sad" vs. "Mark will rapidly go from euphoric to utterly depressed"). The fact that modulations of this sort cannot be reproduced with standard lexical items militates quite strongly

as we have repeatedly made clear, the issue at stake here is the relationship between semantic content and specialized conventions of semantic character, rather than the relationship between semantic content and "general" conventions of meaning (e.g., the kind of non-linguistic conventions explored by recent work on pictorial representation). The issue whether or not the semantic content of presentations of unattested words is constituted by an underlying augmentation of the body of specialized conventions holding in the language, is independent on whether their content is also (partly) dependent on non-specialized conventions of meaning (e.g., in (12), a conventional mapping from decreases in pitch to decreases in tone or affect). Willingness to grant the latter claim would not settle the former. Thanks to two anonymous reviewers for helpful discussion on these points. 
against the idea that [u]-SOUND DESCENDING IN PITCH" ${ }_{(12)}$ and HAND WITH PALM UP + ARM DESCENDING DIAGONALLY $_{(13)}$ may be reduced to modes of non-phonological externalization of "standard" lexical words.

The second reaction would be to accept the comparison but object to the notion that the nonlexical nature of $[\mathrm{u}]$-SOUND DESCENDING IN PITCH" ${ }_{(12)}$ and HAND WITH PALM UP + ARM DESCENDING DIAGONALLY $_{(13)}$ make them unsuited to be captured by Lexical Adaptation. What the comparison shows, if anything, isn't that the content instantiated by novel iconic pseudowords and pro-speech gestures is a counterexample to Lexical Adaptation, but that we should consider an inclusive theory on which the specialized conventions of semantic character that bear on our argument, are not restricted by design to the conventions of "standard" lexical expressions. In other words, linguistic conventions do not have to concern exclusively expressions bearing a phonographic form, but can encompass also pseudowords and gestures, which, when the appropriate requirements are in place, are to be regarded as a genuine lexical objects. As soon as this "accommodating" approach is taken on board, the presentations of (12) and (13) become perfectly amenable to an explanation in terms of Lexical Adaptation: they trigger the transition to a state of the linguistic grammar featuring appropriate conventions of standing linguistic meaning for the gesture and the pseudoword, which turn endow their presentations with semantic content.

Once again, we can grant that this rejoinder would neutralize the pressure. But skepticism seems the most appropriate response to the move. There certainly is a "loose" sense in which we can regard gestures and pseudowords as lexical entities, since in our everyday linguistic life we regularly communicate by combining lexical production with gestural production and other forms of non-linguistic signaling. (12) and (13) wouldn't sound so natural otherwise. It is also fairly evident that it is hard to impose principled restrictions on the kind of forms or shapes that can be registered in the lexical system of language (think of languages where tone is a semantically contrastive feature), and that a language whose lexicon comprises, e.g., gestural elements is perfectly conceivable. However, this seems insufficient to justify the rejoinder in the specific case of (12) and (13). Even if the pseudoword and the gesture were associated to a conventional semantic function, it seems safe to predict that that association would not be registered into the specialized body of semantic conventions that sustains our knowledge of linguistic character. They would end up into a separate non-linguistic inventory (e.g., a gestural lexicon) situated outside the proper boundaries of the linguistic grammar. Absent a clear argument to the contrary, the force of the comparison remains.

The third reaction would be to object to the consistency of the comparison. Precisely because (1) and (11) contain a novel lexical occurrence, whereas the salient constituents of (12) and (13) are 
non-lexical, it would be unfair to imply that because Lexical Adaptation shows no particular promise in capturing (12) and (13), then it should be ruled out as a palatable explanans for the instantiation of semantic content by "shaq" ${ }_{(1)}$ and "schwoom" ${ }_{(11)}$. (12) and (13) cast doubt on Lexical Adaptation only on the premise that its account of the constitution of semantic content can be justifiably asked to apply, besides to (1), also to the properties of "peripheral" presentations such as iconic gestures and iconic pseudowords. Granted, the pseudoword and the gesture of (12) and (13) do fit the label "word-level sentential constituents" featuring in Dynamic L-Conventionalism. But the parallel is problematic. While we found reasons to believe that the lexical innovations featuring in (1) and (11) instantiate semantic content, non-lexical innovations like those observed in (12) and (13) are bound to make a non-semantic contribution to the content of the respective sentences, since they are impossible parts of English.

And yet, recall the toy diagnostics for the instantiation of semantic content we considered in (7)-(10). The argument was that because "shaq" (1) satisfies the diagnostics, the novel zero-derivation complies with Semanticity and instantiates semantic content. And since we have prior motivations to believe that the instantiation of semantic content at the level of word-sized sentential parts has to be grounded on a convention of lexical character, we should buy into Dynamic L-Conventionalism and appeal to Lexical Adaptation. However, if we test (12) and (13) against the same heuristics, it turns out that $[\mathrm{u}]$-SOUND DESCENDING IN $\mathrm{PITCH}_{(12)}$ and HAND WITH PALM UP + ARM DESCENDING $\operatorname{DIAGONALLY}_{(13)}$ satisfy it as well. Both [u]-SOUND DESCENDING IN PITCH $_{(12)}$ and HAND WITH PALM UP + ARM DESCENDING DIAGONALLY ${ }_{(13)}$ do not trigger judgments of infelicity or ungrammaticality, and seem to interact with the grammatical structure of the respective sentences under the standard principles of compositionality. For comparison, see (14), which manages to introduce an antecedent for modal anaphora, (15), which successfully embeds the whistle under negation, and (16), where quantification is perfectly in order.

14) Had Bill kept allowing his mood to [iconic pseudoword: LONG DESCENDING WHISTLE], Lucy would have left him.

15) Bill's mood did not [iconic pseudoword: LONG DESCENDING WHISTLE]. But he wasn't in his best shape either.

16) Whenever somebody wears Bill's magic hat, everybody else's mood [iconic pseudoword: LONG ASCENDING WHISTLE].

In short, if we test (12) and (13) against the same criteria we relied on to argue that the zeroderivation of (1) instantiates semantic content, the pseudowords and the gesture of (12) and (13) 
turn out to comply with Semanticity as well. ${ }^{17}$ However, it remains antecedently clear that the mechanisms whereby these constituents may be endowed with semantic content are unlikely to rely on any prior adaptation of the body of character-level conventions of the language. (12) and (13) are thus direct counterexamples to the thesis that the instantiation of word-level semantic content has to presuppose the existence of an appropriate convention of character in the semantic resources of a language. Absent this bridging posit, there is no implication between the premise that presentations of unattested word-level expressions bear semantic content and participate in sentential composition, and the idea that those features should be analyzed within a framework appealing to the metasemantics of Lexical Adaptation.

This is the relevant lesson we can learn from (12) and (13), and this is why the parallel between (1) and the realm of onomatopoeic words, iconic pseudowords, and gestures matters to our discussion: the "critical juncture" we mentioned earlier and identified as the cornerstone of the case for Lexical Adaptation, is a dispensable requirement. We can, it seems, discount the principle that word-level sentential constituents bearing full-blooded semantic content (the kind of content that can feed the compositional matrix of a linguistic sentence) have to do so as a function of background linguistic character; reconsider the grounds for thinking that our verdict on (1) should not deviate dramatically from L-Conventionalism; and revisit our metasemantic assumptions based on the independent evidence provided by cases like (12) and (13). The word-level constituents of a sentence can instantiate semantically efficacious content even if the body of conventions available to the language are silent, or are bound to remain silent (when the word-level parts concerned are non-linguistic), about their standing meaning. It is not necessarily the case that either the novel zero-derivation of (1) bears semantic content relative to an underlying update in the character-level conventions of the language, or leaves a semantic gap in the sentence. The comparison with (12) and (13) paves the way for a different diagnosis: assume that (1) satisfies Semanticity, grant that “shaq" (1) bears semantic content, and put L-Conventionalism on hold with a theory on which the word-level constituents of a sentence can be endowed with semantic, compositionally efficacious content without such content being the output of a function from standing linguistic meaning.

17 One might object that this conclusion is a figment of the limited battery of tests we have considered, or that a more careful heuristics might do the job of disentangling the innovation of (1) from the non-linguistic constituents featuring in (12) and (13). However, there is growing independent consensus that iconic constituents like [u]-SOUND DESCENDING IN PITCH $(12)$ and HAND WITH PALM UP + ARM DESCENDING DIAGONALLY(13) do trigger sophisticated semantic and compositional effects, and that they do so even on first exposure. Besides entailments, they seem to trigger scalar implicatures, presuppositions and associated phenomena (e.g., anti-presuppositions), homogeneity inferences characteristic of definite plurals, as well as some expressive inferences normally found in pejorative terms. See, a.o., Abush (2012), Ebert and Ebert (2014), and Schlenker (2019). 


\section{Productive Periphery}

Now that we have all the ingredients on the table, we can lay out our alternative take on the problem. We have already anticipated the central features of the recipe, the task is now to give it a more orderly formulation. (1) satisfies Semanticity: "shaq" ${ }_{(1)}$ bears semantic content and the sentence is not compositionally gappy at the position occupied by the zero-derivation, despite the absence of an entry specifying the conventional meaning of $s h a q_{\mathrm{v}}$ in the lexicon of the language. However, the instantiation of semantic content on behalf of the unfamiliar presentation is not grounded on an underlying transition to an augmented variant of English featuring a norm of standing meaning for $s h a q_{\mathrm{v}}$. Instead, we should entertain the hypothesis that the instantiation of semantic content by "shaq" (1) follows the same metasemantic blueprint suggested by presentations of novel onomatopoeic words (e.g., "schwoom" ${ }_{(11)}$ ), novel iconic pseudowords (e.g., [u]-SOUND DESCENDING IN PITCH $(12)$ ), and novel pro-speech gestures (e.g., HAND WITH PALM UP + ARM DESCENDING DIAGONALLY(13)).

The claim is twofold. First, observation of the semantic properties instantiated by the inaugural presentations of novel iconic constituents gives reason to withdraw from the rule that the instantiation of specialized, semantic, compositionally efficacious content presupposes standing linguistic meaning. Second, we can use this revised metasemantics to propose that the correct solution to the tension between Semanticity and L-Conventionalism is to dispense with LConventionalism. On the resulting view, "shaq" ${ }_{(1)}$ receives compositionally efficacious content as a result of the combined pressure of facts about the style of play of Shaquille O'Neal, facts about the observable morphosyntactic features of the unfamiliar word, facts about sentential context, facts about the subject matter of the conversation, and so forth. The joint normative force exerted by these facts from the periphery of the grammar suffices to endow the novel zero-derivation with the entire set of semantic features the unfamiliar word needs to comply with Semanticity. Hence, instead of Lexical Adaptation, we should have what follows.

\section{Productive Periphery}

i. $\quad$ The speaker produces (1) and "shaq" (1) is inaugurally presented.

ii. The presentation of (1) occurs in a situation where a set $\mathrm{F}$ of non-semantic facts about "shaq" ${ }_{(1)}$ holds: that the presentation exhibits a certain set of inflectional features, that it is derived from the name of Shaquille O'Neal, that the sentence where it occurs is describing a basketball play, that Shaquille O'Neal is stereotypical associated with a particular style of play, and so forth. 
iii. The facts in F generate the semantic content instantiated by "shaq"

The departure from Lexical Adaptation is clear enough. On Lexical Adaptation, the presentation of (1) triggers the transition to a state of the language featuring a proto-convention about the standing properties of $s h a q_{\mathrm{v}}$, and "shaq" ${ }_{(1)}$ bears semantic content relative to this underlying augmentation of the specialized semantic resources of the language. On the account I am encouraging to consider, the instantiation of semantic-compositional content by "shaq" (1) is not mediated by any vocabulary-level middle man: it is generated directly by the combined pressure of the set of non-semantic facts surrounding the presentation of (1). On the proposed view, the lesson we should learn from the tension between Semanticity and L-Conventionalism created by (1) is not that we should dynamicize L-Conventionalism (irrespective of the fact that thinking about linguistic conventions as dynamic objects might be independently useful and entirely correct); rather, we should shift away from L-Conventionalism and allow for a metasemantics where non-linguistic facts can exert grammatical efficacy, and generate semantic content and compositional roles for word-sized expressions, without the prior intervention of a function from character.

Of course, the account is fairly programmatic (just as programmatic as Lexical Adaptation itself), and leaves important questions open. What are the specifics of the mechanisms whereby the non-semantic facts surrounding the presentation of an unfamiliar word can bring about its instantiation of semantic content? How exactly does the interplay of facts about Shaquille O'Neal, facts about the morphosyntactic features of "shaq" ${ }_{(1)}$, facts about the goals of the conversational exchange, and so forth, manage to result in the determination of semantic content for "shaq" (1)? How does Productive Periphery fit with known constraints on lexical innovation, such as lexical contrast (Clark 1993) or the resistance to the coinage of new function words? What is the relationship between everything we have said so far and the speaker's intention in producing (1), and, in particular, do we want speaker intentions to feature in the set of non-semantic facts that bring about semantic content?

These are all open questions, but nothing in Productive Periphery seem to stand in the way of giving them good answers. Suppose, for the sake of argument, we answer the last question in the negative; intentions are an intricate business and we want to exclude them from the landscape of facts that determine semantic content in a case like (1). ${ }^{18}$ Suppose, further, (1) is inaugurally uttered by a speaker who is confused about Shaquille O'Neal and produces the zero-derivation with the intention to mean a style of play Shaq never pursued in his decorated basketball career. This would

18 For the record, I am considering the possibility simply to illustrate the neutrality of Productive Periphery, not to imply that we should go for intention-insensitivity. On intentions and conventions, see, e.g., Schiffer (2017). 
seem to lead to the problematic conclusion that speakers can be confused about the meaning of the very lexical innovation they are coining. But I don't think this conclusion is as problematic as it might look at first glance. Externalist intuitions can come to the rescue. A speaker confused about water and producing utterances of the noun water $_{\mathrm{N}}$ with the intention to refer to XYZ (or with the belief that water $_{\mathrm{N}}$ refers to XYZ) has no jurisdiction over the norm that utterances of water $_{\mathrm{N}}$ in English pick out $\mathrm{H}_{2} \mathrm{O}$. Similarly, a speaker confused about Shaquille O'Neal uttering (1) with the intention to refer to a style of play having nothing to do with Shaq may have no control over the meaning that the verb is bound to instantiate given the non-semantic facts that surround it.

Similarly, consider the issue of establishing in what way Productive Periphery may do justice to the intuition that the presentation of (1) establishes a lexical precedent that can undergo fullfledged conventionalization. Again, nothing in the proposed account makes it especially problematic to produce a story about conventionalization, and it is far from clear that a viable theory of how full-fledged coordination on the conventional meaning of a new content word is achieved within a group of speakers, should rely on the analysis of (1) given by Lexical Adaptation. Lexical Adaptation argues that the birth of a proto-convention of standing meaning for $s h a q_{\mathrm{v}}$ is intrinsic to the instantiation of semantic content by "shaq" ${ }_{(1)}$. On Productive Periphery, the birth of the proto-convention is not intrinsic to the instantiation of semantic content by "shaq" (1), but can obviously occur as a result of the presentation of the novel word to the linguistic community and, where applicable, of subsequent collective bargaining on the linguistic roles the term should play in the language.

One last comment about the comparison between (1) and (11)-(13). I have proposed that the parallel with (11)-(13) suggests an argument to give up L-Conventionalism in theorizing about the grounds of (1)'s compliance with Semanticity. It bears emphasizing that the suggestion does not, nor is intended to, obliterate the important differences that are inevitably going to be found between the psychological processes which govern the interpretation of new iconic words, iconic pseudowords and pro-speech gestures, as opposed to those that allow speakers to interpret "arbitrary" lexical novelties like "shaq", ${ }_{(1)}$ I am not trying to infer, from the case for a uniform metasemantic treatment of these cases, the claim that interpretive algorithms at work in the two domains are identical twins and wipe out the divide between iconic and arbitrary signs. ${ }^{19}$ Quite the

19 Though it might be worth mentioning that linguistic theory has been steadily shifting away from the consensus that while iconicity can be found in spoken languages, phenomena such as onomatopoeia and sound symbolism are “asterisks to the far more important principle of the arbitrary sign" (Pinker 1999: 2). A flurry of findings suggests that iconic form-to-meaning mappings are much more pervasive (in signed and spoken lexicons alike) than the dogma of arbitrariness suggests, and that elements of iconicity tend to be present, in various degrees and at various levels (phonemic, prosodic, morphological), even in the linguistic forms we have learned to characterize as 
contrary: there are going to be considerable differences. The interpretation of iconic gestures relies on an integration of information from the visual modality that is absent in cases of non-iconic lexical novelty. The same goes for pitch with the pseudoword of (12). Similarly, while pro-speech gestures often make it possible to recover a very fine-grained meaning, thanks to their gradient features, listeners exposed to non-iconic lexical innovations are often able to match the newly encountered words with a rather coarse-grained content. Even when elements of sound symbolism are available, as in the case of "schwoom" ${ }_{(11)}$, the granularity of the meaning speakers are able to retrieve on first exposure to the new word may be quite coarse: a fast horizontal movement possibly producing a certain type of sound. Another difference is that while the reconstruction of the action designated by "schwoom" ${ }_{(11)}$ rests on context plus an iconic analysis of the shape of the verb, the interpretation of "shaq" ${ }_{(1)}$ requires context and a more complex plausibility inference. The listener has to determine what properties, among those associated to Shaquille O'Neal, provide the correct blueprint for the interpretation of the verb (physical dominance and style of play as opposed to having an eccentric media personality). ${ }^{20}$ The proposal I am making accepts these differences, but invites to consider a broader point of potential metasemantic commonality holding across the spectrum of these cases: in essence, the ability to be invested with compositionally efficacious content as a result of facts sitting entirely outside the standing semantic resources of the language.

\section{Conclusion}

The discussion has proceeded as follows. Section 1 introduced Semanticity and LConventionalism, and described the tension surfacing when their combination is tested against sentences featuring lexical innovations. Section 2 presented the view that lexical innovations bear semantic content as a result of an underlying update of the conventions of standing meaning holding in the language. Section 3 reexamined the case for the dynamic-conventionalist solution and, building on a comparison with the production of novel onomatopoeic words, iconic pseudowords and pro-speech gestures, drew attention to the possibility of an alternative analysis of the tension. Section 4 laid out the alternative analysis: the view that the semantic-compositional properties instantiated by presentations of unfamiliar words motivate a metasemantic allowing word-sized

“arbitrary”. See, e.g., Hinton, Nichols and Ohala (2006); Perniss, Thompson and Vigliocco (2010).

20 Besides, recall that the properties of the shape [ wum] are insufficient to fix any univocal meaning and that, much like in (1), reasoning about context has to pull its weight. If the inaugural presentation of "schwoom", instead of occurring in (11), occurred in the sentence "The stocks were going up yesterday but today they'll most certainly schwoom", the verb would be readily understood to denote something very different from the event pictured by "schwoom" ${ }_{(11)}$ (presumably, a fall instead of a swift horizontal movement). So (1) and (11) are not as distant as the difference in iconic character might seem to suggest. 
constituents to be endowed with semantic content directly as a result of the set of non-semantic facts surrounding the presentations at stake. The take-home message is the following: we should consider an approach to lexical innovations that does not foresee any necessary or constitutive role for linguistic conventions among the factors that endow inaugural presentations of unattested words with semantic content. If this is correct, dynamic maneuvers do not help rescue L-Conventionalism from the problem of lexical innovation.

Where does this leave us with respect to long-standing debates about the conventional nature of linguistic meaning? I would offer two final comments. The first is about the implications of the argument on the analysis of "regular" or non-innovative language. Productive Periphery is committed to the idea that our resolution of the tension surfacing from (1) should not aim to keep LConventionalism in the game. However, the resulting brand of anti-conventionalism should be interpreted with sufficient nuance. The suspension of L-Conventionalism encouraged by Productive Periphery is in not necessarily enemy to the proposition that in cases involving fully conventionalized words, the instantiation of semantic content is successfully characterized by LConventionalism, and accordingly that the principle captures the "normal" modus operandi of semantic content. Davidson and a line of subsequent commentators have certainly assumed that the fate of conventionalist approaches to meaning hinged on their ability to generalize to cases of lexical innovation. Should they fail to illuminate the interpretive dynamics in play on first exposure to unfamiliar words, the conclusion to draw would be that conventionalism is false, and that linguistic conventions do not provide explanatory purchase on issues of semantic content (for considerations in this spirit, see Lepore and Stone 2017). I believe we should consider resisting "totalitarian" assumptions of this sort. For example, L-Conventionalism might remain a sound principle to hold for sentences and constituents built on familiar linguistic material even if none of its incarnations can hope to adequately account for the semantic effects triggered by lexical innovations. Consider the following.

L-Conventionalism* The semantic content of attested word-level sentential constituents is a function of the standing lexical conventions of the language.

Modulo a working definition of "attested", the conjunction of L-Conventionalism* and Semanticity seems a perfectly viable way of accounting for the semantic properties of the vast realm of everyday linguistic production not involving the presentation of unfamiliar words. ${ }^{21}$ As long as the line we

21 Another way to put the point: proof that L-Conventionalism is universally false does not make it generically false. If I am correct, universal interpretations of L-Conventionalism are refuted by the negative instances provided by 
seem able to draw between "standard" and "innovative" linguistic production tracks two distinct facets or spheres of the life of a language, each responding to potentially distinct principles and requiring potentially distinct metasemantic frameworks, we should be careful inferring, from the difficult predicament faced by L-Conventionalism in the realm of lexical innovations, any harm to its validity for the run-of-the-mill aspects of language it was originally designed to capture.

The second comment is about the prospects of a "general" conventionalist approach to the semantics of lexical innovation. As I have repeatedly made clear, my argument has been dealing with a specific incarnation of the conventionalist dogma, and has centered on the interplay of semantic content and specialized linguistic conventions of semantic character. I have argued that the rejection of L-Conventionalism is consistent with the belief in a necessary role for non-linguistic conventions (e.g., social conventions) in the determination of levels of linguistic meaning such as pragmatically enriched utterance meaning. Let me conclude with noting that Productive Periphery is also consistent with the idea that non-linguistic conventions may be indispensable to the constitution of the zero-shot semantic content of lexical innovations themselves. As I hope to have been able to illustrate, the issue whether L-Conventionalism is able to respond to the pressure from lexical innovation is intricate enough and rich enough in implications to warrant autonomous investigation. I have made my case that our verdict in this specific regard should be Davidsonian and anti-conventionalist. But would be improper to characterize the resulting view as the claim that our approach to the semantics of lexical innovation should be unrestrictedly anti-conventionalist. Productive Periphery warrants no negative stance on the claim that the presentations of unattested words manage to instantiate semantic content only through the mediation of some suitable body of general non-linguistic conventions. Personally, I find unrestricted anti-conventionalism hard to swallow as a general theory of meaning, and I would be surprised if the negative verdict on LConventionalism reached by Productive Periphery triggered a cascade effect on all kinds of conventions. But that's a topic for another occasion.

cases of lexical innovation. Nonetheless, L-Conventionalism might remain sound as a generic claim, that is, as a claim about the factors that endow presentations of lexical expressions with semantic content in "typical" cases, and modulo the premise that utterances of sentences built exclusively with familiar lexical material are the statistic norm. Compare with Armstrong (2015) on "moderate explanatory conventionalism". 


\section{References}

Abusch, D. (2012). Applying discourse semantics and pragmatics to co-reference in picture sequences. Proceedings of Sinn und Bedeutung 17: 9-25.

Armstrong, J. (2015). Coordination, triangulation, and language use. Inquiry 59: 80-112.

Armstrong, J. (2016). The problem of lexical innovation. Linguistics and Philosophy 39: 87-118.

Ball, D., \& Rabern, B. (eds.) (2018). The Science of Meaning: Essays on the Metatheory of Natural Language Semantics. Oxford: Oxford University Press.

Barber, A. (2001). Idiolectal error. Mind \& Language 16: 263-283.

Bauer, L. (2001). Morphological Productivity. Cambridge: Cambridge University Press.

Borg, E. (2004). Minimal Semantics. Oxford: Oxford University Press.

Burgess, A., \& Sherman, B. (eds.) (2014). Metasemantics: New Essays on the Foundations of Meaning. New York, NY: Oxford University Press.

Bybee, J. (2015). Language Change. Cambridge: Cambridge University Press.

Clark, E. (1993). The Lexicon in Acquisition. Cambridge: Cambridge University Press.

Cumming, S., Greenberg, G., \& Kelly, R. (2017). Conventions of viewpoint coherence in film. Philosophers'Imprint 17 (1): 1-29.

Davidson, D. (1984). Convention and communication. Synthese 59: 3-17.

Davidson, D. (1986). A nice derangement of epitaphs. In R. Grandy \& R. Warner (eds.), The Philosophical Grounds of Rationality. 443-446. Oxford: Oxford University Press.

Davidson, D. (1991). James Joyce and Humpty Dumpty. Midwest Studies In Philosophy 16: 1-12.

Dowty, D. (1979). Word Meaning and Montague Grammar. Dordrecht: Kluwer.

Ebert, Co., \& Ebert, Ch. (2014). Gestures, demonstratives, and the attributive/referential distinction. Handout of a talk given at Semantics and Philosophy in Europe (SPE 7), Berlin, June 28 ${ }^{\text {th }}$, 2014. https://semanticsarchive.net/Archive/GJjYzkwN/EbertEbert-SPE-2014-slides.pdf

Elman, J. L. (2009). On the meaning of words and dinosaur bones: lexical knowledge without a lexicon. Cognitive Science 33: 547-582.

Fromkin, V. (Ed.) (2000). Linguistics: An Introduction to Linguistic Theory. Malden, MA: Blackwell.

Gasparri, L., \& Murez, M. (2019). Hearing meanings: the revenge of context. Synthese. https://doi.org/10.1007/s11229-019-02398-0.

Greenberg, G. (2013). Beyond resemblance. Philosophical Review 122: 215-287.

Halle, M., \& Marantz, A. (1993). Distributed Morphology and the pieces of inflection. In K. Hale \& S. J. Keyser (eds.), The View from Building 20: Essays in Linguistics in Honor of Sylvain Bromberger. 111-176. Cambridge, MA: MIT Press. 
Halle, M., \& Marantz, A. (1994). Some key features of Distributed Morphology. MIT Working Papers in Linguistics 21: 275-288.

Higginbotham, J. (1986). Linguistic theory and Davidson's program in semantics. In E. Lepore (ed.), Truth and Interpretation: Perspectives on the Philosophy of Donald Davidson. 29-48. New York: Blackwell.

Hinton, L., Nichols, J., \& Ohala, J. J. (eds.) (2006). Sound Symbolism. Cambridge: Cambridge University Press.

Jackendoff, R. (2002). Foundations of Language: Brain, Meaning, Grammar, Evolution. Oxford: Oxford University Press.

Kaplan, D. (1989). Demonstratives. In J. Almog, J. Perry, \& H. Wettstein (eds.), Themes from Kaplan. 481-563. New York: Oxford University Press.

Kendon, A. (2004). Gesture: Visible Action as Utterance. Cambridge: Cambridge University Press.

Larson, R., and G. Segal. (1995). Knowledge of Meaning: An Introduction to Semantic Theory. Cambridge, MA: MIT Press.

Lascarides, A., and M. Stone. (2009). A formal semantic analysis of gesture. Journal of Semantics 26: 393-449.

Lepore, E., and M. Stone. (2017). Convention before communication. Philosophical Perspectives 31: $245-265$.

Lewis, D. K. (1969). Convention: A Philosophical Study. Cambridge, MA: Harvard University Press.

Ludlow, P. (2014). Living Words: Meaning Underdetermination and the Dynamic Lexicon. New York: Oxford University Press.

MacFarlane, J. (2014). Assessment Sensitivity: Relative Truth and its Applications. Oxford: Oxford University Press.

Perniss, P., Thompson, E. L., \& Vigliocco, G. (2010). Iconicity as a general property of language: Evidence from spoken and signed languages. Frontiers in Psychology 1: Article 227. https://doi.org/10.3389/fpsyg.2010.00227.

Pinker, S. (1999). Words and Rules: The Ingredients of Language. London: Weidenfeld \& Nicolson.

Plunkett, D., \& Sundell, T. (2013). Disagreement and the semantics of normative and evaluative terms. Philosophers' Imprint 13 (23): 1-37.

Predelli, S. (2010). Malapropisms and the simple picture of communication. Mind \& Language 25: 329-345.

Rayo, A. (2013). A plea for semantic localism. Nô̂s 47: 647-679.

Recanati, F. (2010). Truth-Conditional Pragmatics. Oxford: Oxford University Press. 
Schiffer, S. (2017). Intention and convention in the theory of meaning. In B. Hale, C. Wright \& A. Miller (eds.), A Companion to the Philosophy of Language, $2^{\text {nd }}$ edn. 49-72. Malden, MA: Wiley.

Schlenker, P. (2018). Gesture projection and cosuppositions. Linguistics and Philosophy 41: 295365.

Schlenker, P. (2019). Gestural semantics. Natural Language \& Linguistic Theory 37: 735-784.

Szabó, Z. (ed.) (2005). Semantics versus Pragmatics. Oxford: Clarendon Press.

Xian, Y., Lampert, C. H., Schiele, B., \& Akata, Z. (2018). Zero-shot learning: A comprehensive evaluation of the good, the bad and the ugly. https://arxiv.org/abs/1707.00600v3. 